\title{
Clinical Characteristics and Transmission Routes of COVID-19 in the Early Period of the Pandemic in a Non-Covid Ward of Chest Diseases Hospital
}

\section{Pandeminin Erken Döneminde Göğüs Hastalıkları Hastanesinin Covid Dışı Bir Servisinde COVID-19'un Klinik Özellikleri ve Bulaşma Yolları}

\author{
Mine GAYAF $\odot$, Ceyda ANAR $\oplus$, Filiz GüldDAVAL $\oplus$, Gülistan KARADENiz $\oplus$, Gülru POLAT $\odot$ \\ Aysu AYRANCI $\odot$, Özgür BATUM $\odot$, Dursun TATAR $\odot$
}

Cite as: Gayaf M, Anar C, Güldaval F, Karadeniz G, Polat G, Ayrancı A, et al. Clinical characteristics and transmission routes of COVID-19 in the early period of the pandemic in a non-covid ward of a chest diseases hospital. İzmir Göğüs Hastanesi Dergisi. 2021;35(3):140-8.

\section{ABSTRACT}

Objective: In the early stages of the outbreak, Covid patients were followed-up in isolated Covid Wards, and the examinations of other pulmonary diseases continued in Non-Covid wards. Differential diagnosis between COVID-19 and infections caused by other pathogens is not adequately recognized. For this reason, it is even more difficult to identify patients who are infected with SARS-CoV2 or other pathogens. In the present study, the clinical characteristics and transmission routes of the Covid-19 cases in Non-Covid Wards within approximately 2 months' time after the onset of the pandemic were analyzed speculatively.

Method: In the early periods of the pandemic, quarantine wards were created for Covid patients in Chest Diseases Hospital, but there were also changes in Non-Covid patients and new Non-Covid hospitalizations. The clinical characteristics and transmission routes of the Covid cas

observationally between 10.03 .2020 and 30.04 .2020 . Results: During this period, a total of 35 Covid cases were detected as a patient, companion and healthcare employees. The median age of the patients was 50 years ( $\min -\max 25-85)$. There were 17 patients $(48.6 \%)$ who were not severe, and 18 severe patients $(51.4 \%)$, and $10(28.6 \%)$ patients died. A total of $25(71,4 \%)$ of infected cases were verified with the SARS-CoV-2 Nucleic Acid Test, and $10(28,6 \%)$ were diagnosed clinically and radiologically as possible COVID-19 cases. Being 65 years old or over, having comorbid diseases, especially COPD, the presence of dyspnea as a symptom and involvement on chest radiography were found to be significantly associated with survival (p0.027, $0.009,0.038,0.000$ and 0.033 , respectively). Lymphopenia, increase in neutrophil count, CRP and NLR value were found to be statistically related to survival ( $0.005,0.001,0.014$ and 0.000 , respectively). We found 4 super spreaders, one of whom was a companion, and 3 patients.

Conclusion: Potential "super spreaders" can be the source of infection before the quarantine conditions are applied and comprehensive protection is implemented. For this reason, quarantine, use of personal protective equipment, application of social distancing, and the implementation of comprehensive preventive measures, such as disinfection, are crucial in controlling nosocomial infection.

Keywords: COViD-19, transmission, pandemic

ÖZ

Amaç: Salgının erken evrelerinde Covid hastaları izole COVID Servislerinde takibe alınırken, diğer akciğer hastalıklarının tetkiklerine COViD olmayan servislerde devam edildi. COVID-19 ile diğer patojenlerin neden olduğu enfeksiyonlar arasındaki ayırıcı tanı yeterince tanınmamaktadır. Bu nedenle SARS-CoV2 veya başka bir patojen ile enfekte olan hastaları tespit etmek daha da zorlaşıyor. Bu çalıșmada, Covid-19 vakalarının yaklașık 2 ay içinde Covid- 19 dıșı servislerdeki klinik özellikleri ve bulașma yolları incelendi. Pandeminin bașlangıcından sonraki süre spekülatif olarak analiz edildi.

Yöntem: Pandeminin erken dönemlerinde Göğüs Hastalıkları Hastanesinde Covid hastaları için karantina koğușları olușturulurken Covid olmayan hastalarda ve yeni Covid dıșı yatıs̆larda da değișiklikler oldu. 10.03.2020 ile 30.04.2020 tarihleri arasında Kovid Dışı Serviste bulunan Covid vakalarının klinik özellikleri ve bulaşma yolları geriye dönük ve gözlemsel olarak incelendi.

Bulgular: Bu dönemde hasta, refakatçi ve sağlık çalışanı olmak üzere toplam 35 Covid vakası tespit edildi. Hastaların ortanca yașı 50 (min-maks $25-85)$ idi. Ağır olmayan 17 hasta $(\% 48,6), 18$ ağır hasta $(\% 51,4)$ ve 10 hasta öldü $(\% 28,6)$. Toplam 25(\%71,4) enfekte vaka SARS-CoV-2 Nükleik Asit Testi ile doğrulandı ve $11(\% 28,6)$ olası COVID-19 vakas olarak klinik ve radyolojik olarak teşhis edildi. 65 yaș ve üzeri olmak, KOAH bașta olmak üzere komorbid hastalığı olmak, semptom olarak nefes darlı̆̆ın varlı̆̆ ve akciğer grafisinde tutulum sağkalım ile anlamlı olarak ilişkili bulundu (sirasiyla p0.027, 0.009, 0.038, 0.000 ve 0.033). Lenfop (1) sağkalım ile istatistiksel olarak iliskili bulundu (sırasıyla p $0,005,0,001,0,014$, 3 hasta bulundu.

Sonuç: Potansiyel "süper yayıcılar”, karantina koşulları uygulanmadan ve kapsamlı koruma uygulanmadan önce enfeksiyon kaynağı olabilir. Bu nedenle karantina, kişsisel koruyucu ekipman kullanımı, sosyal mesafenin uygulanması ve dezenfeksiyon gibi kapsamlı önleyici tedbirlerin uygulanması hastane enfeksiyonunun kontrolünde çok önemlidir.

Anahtar kelimeler: COViD-19, bulaşma, pandem
Received/Gelis: 05.08.2021

Accepted/Kabul: 03.09.2021

First Published/Erken Cevrimiçi: 24.09.2021

Corresponding author/Sorumlu yazar:

C. Anar

ORCID: 0000-0002-3922-5800 İzmir Katip Celebi Üniversitesi Atatürk Eğitim Araştırma Hastanesi, Göğüs Hastalıkları Bölümü İzmir - Türkiye

drceydaanar@hotmail.com

M. Gayaf

ORCID: 0000-0003-1375-6221

F. Güldaval

ORCID: 0000-0002-7712-5093

G. Karadeniz

ORCID: 0000-0002-1994-6723

G. Polat

ORCID: 0000-0002-2211-1268

Ö. Batum

ORCID: 0000-0002-8353-3421

D. Tatar

ORCID: 0000-0002-6285-3549

SBÜ. Dr. Suat Seren Göğüs Hastalıkları ve Cerrahisi Eğitim Araştırma Hastanesi, Göğüs Hastalıkları Bölümü İzmir - Türkiye

A. Ayrancı

ORCID: 0000-0002-8939-336X İzmir Bakırçay Üniversitesi Çiğli Ĕgitim ve Araştırma Hastanesi, Göğüs Hastalıkları Kliniği, İzmir, Türkiye

() Telif hakkı İzmir Göğüs Hastanesi'ne aittir. Logos Tıp Yayıncllık tarafından yayınlanmaktadır.

Bu dergide yayınlanan bütün makaleler Creative Commons 4.0 Uluslararası Lisansı (CC BY) ile lisanslanmıștır. 
M. Gayaf ve ark., Clinical Characteristics and Transmission Routes of COVID-19 in the Early Period of the Pandemic in a Non-Covid Ward of a Chest Diseases Hospital

\section{INTRODUCTION}

More than 80.000 .000 people were infected with SARS-CoV-2 resulting in many deaths in 60 countries all over the world since the Corona Virus Disease (COVID-19) appeared in Wuhan in December 2019. All age groups are sensitive to SARS-CoV-2 infection. The infection routes are airborne and with close contact. The fecal-oral route and aerosol infection cannot be excluded. SARS-CoV-2 infection outbreaks at hospitals appeared in early periods of the outbreak, before the disease was noticed and before quarantine measures were put into practice. Differential diagnosis regarding COVID-19 and infections of other pathogens was not adequately recognized in the early stages of the outbreak. For this reason, it is more challenging to identify patients who are infected with SARS-CoV2, and to take preventive measures to avoid nosocomial infection. In the present study, we analyzed the clinical features and transmission routes of 35 COVID-19 cases from a single Chest Diseases Department, including 16 patients (one of them is a medical companion) and 19 medical staff in non-Covid services.

\section{MATERIALS and METHOD}

\section{Study Population}

In the early periods of the pandemic, quarantine wards were created for Covid patients in Chest Diseases Hospital, but there were also changes in Non-Covid patients and new Non-Covid hospitalizations. The clinical characteristics and transmission routes of the Covid cases in NonCovid Ward were examined retrospectively and observationally between 10.03.2020 and 30.04.2020.

A total of 35 cases with the epidemiological examination, laboratory results, computed tomography imaging (CT), and SARS-CoV-2 Nucleic Acid Test results were included in the study of 16 hospitalized patients (3 of them medical companion) and 19 medical staff. The study was approved by Medical Ethics Committee of Izmir Dr. Suat Seren Chest Disease Training and Research Hospital, Turkey. Also, Helsinki Declaration was approved.

\section{Data Collection}

Clinical medical records were used to obtain the clinical data of COVID-19 cases. Fever and CT imaging characteristics of all cases were evaluated, and SARS-CoV-2 Nucleic Acid Tests were done for suspected individuals. All infected medical staff were interviewed about the duration of exposure to the COVID-19 cases, personal preventive measures, and clinical symptoms. All COVID-19 cases were monitored. The data of the patients included gender, age, comorbid disease, smoking history, contact history, fever, and laboratory examination results in treatment, SARS-CoV-2 Nucleic Acid Test results, CT imaging, and the condition during the latest follow-up date. The most likely infection time and route were speculatively examined and estimated according to the history of exposure in the ward, evidence of infection, and the status of inter-ward transfer.

\section{Clinical Classification of COVID-19}

The cases were classified according to symptoms, laboratory and radiological findings. The Mild Stage includes mild clinical symptoms, and no pneumonia signs in imaging. The Severe Stage involved fever, respiratory tract, and other symptoms, signs in pneumonia imaging. Severe Stage, where one of the following criteria are present; (a) shortness of breath, respiratory rate $\geq 30$ times/min; (b) during resting, oxygen saturation less than 93\%; and (c) $\mathrm{PaO}_{2} / \mathrm{FiO}_{2} \leq 300$ $\mathrm{mmHg}$, Group with CT imaging in 24-48 hours with a $50 \%$ significant lesion progression ${ }^{(1)}$.

\section{Statistical Analyses}

The quantitative data are given as Mean \pm Standard Deviation (SD), and the Independent Student's t-test was employed in inter-group comparisons. The qualitative data are given as 
case count (percentages), and the groups were compared with Fisher's Exact Test. A p value $<0.05$ was taken as statistically significant.

\section{RESULTS}

\section{Clinical Characteristics}

A total of $11(31.4 \%)$ of the patients were male, and 24 (68.6\%) were female. The median age of the patients was 50 (min-max 25-85) and the median age of the medical staff was 42 (minmax 36-85). There were 17 patients (48.6\%) who were not severe, and 18 severe patients (51.4\%), and $10(28.6 \%)$ patients died. A total of 25 $(71,4 \%)$ of infected cases were verified with the SARS-CoV-2 Nucleic Acid Test, and 10 (28,6\%) were diagnosed clinically and radiologically as possible COVID-19 . A total of 25 (76\%) of the infected cases had a history of positive exposure. Two patients were infected from patient to companion, 4 were infected from medical staff to medical staff, 9 were infected from patient to medical staff, the other 9 were infected from patient to patient, and 1 case was infected from companion to patient (Table 1).

When health care workers (HCW) were compared with patients, the symptoms of additional disease, cough, shortness of breath, and the presence of lesions in the lung $x$-ray were statistically significant in patients who were ill compared to healthcare staff (Table 2). COPD, which is comorbidity, was detected more in COPD patients at a statistically significant level ( $p$ : 0.006) (Table 2).

\section{Prognosis Analysis for COVID-19 Cases}

All of the cases who were infected with SARSCoV-2 were under quarantine and were treated. There were 10 deaths (28.6\%). Being 65 years old or over, having comorbidities, especially COPD, the presence of dyspnea as a symptom and involvement on chest radiography were found to be significantly associated with survival (p:0.027, 0.009, 0.038, 0.000 and 0.033, respectively) (Table 3 ). Lymphopenia, increase in
Table 1. General characteristics of cases

\begin{tabular}{|c|c|c|}
\hline Characteristics & $\mathbf{N}$ & $\%$ \\
\hline $\begin{array}{l}\text { Female } \\
\text { Male }\end{array}$ & $\begin{array}{l}24 \\
11\end{array}$ & $\begin{array}{l}68.6 \\
31.4\end{array}$ \\
\hline $\begin{array}{l}\text { Age } \\
\geq 65 \text { age } \\
<65 \text { age }\end{array}$ & $\begin{array}{l}8 \\
27\end{array}$ & $\begin{array}{l}22.9 \\
77.1\end{array}$ \\
\hline $\begin{array}{l}\text { Personnel } \\
\text { Nurse } \\
\text { Doctor } \\
\text { Patient } \\
\text { Companion }\end{array}$ & $\begin{array}{l}4 \\
10 \\
5 \\
13 \\
3\end{array}$ & $\begin{array}{l}11.4 \\
28.6 \\
14.3 \\
37,1 \\
8.6\end{array}$ \\
\hline $\begin{array}{l}\text { Smoking Status } \\
\text { Smoker } \\
\text { Nonsmoker } \\
\text { Ex-smoker }\end{array}$ & $\begin{array}{l}7 \\
17 \\
11 \\
\end{array}$ & $\begin{array}{l}20 \\
48,6 \\
31.4 \\
\end{array}$ \\
\hline $\begin{array}{l}\text { Comorbidity } \\
\text { Yes } \\
\text { No }\end{array}$ & $\begin{array}{l}18 \\
17\end{array}$ & $\begin{array}{l}51.4 \\
48,6\end{array}$ \\
\hline $\begin{array}{l}\text { Hypertension } \\
\text { Diabetes Mellitus } \\
\text { COPD } \\
\text { KAH } \\
\text { Asthma } \\
\text { Lung Cancer }\end{array}$ & $\begin{array}{l}6 \\
3 \\
6 \\
2 \\
1 \\
2 \\
\end{array}$ & $\begin{array}{l}17.1 \\
8.6 \\
17.1 \\
5.7 \\
2.9 \\
5.7 \\
\end{array}$ \\
\hline $\begin{array}{l}\text { Symptom } \\
\text { Yes } \\
\text { No }\end{array}$ & $\begin{array}{l}29 \\
6 \\
\end{array}$ & $\begin{array}{l}82.9 \\
17.1\end{array}$ \\
\hline $\begin{array}{l}\text { Fever }(\geq 37.3) \\
\text { Cough } \\
\text { Myalgia } \\
\text { Sore throat } \\
\text { Shortness of breath } \\
\text { Diarrhea }\end{array}$ & $\begin{array}{l}16 \\
20 \\
16 \\
9 \\
15 \\
3 \\
\end{array}$ & $\begin{array}{l}45.7 \\
57.1 \\
45.7 \\
25.7 \\
42.9 \\
8.6 \\
\end{array}$ \\
\hline $\begin{array}{l}\text { Disease Severity } \\
\text { Mild } \\
\text { Severe }\end{array}$ & $\begin{array}{l}17 \\
18\end{array}$ & $\begin{array}{l}48,6 \\
51,4\end{array}$ \\
\hline $\begin{array}{l}\text { Exposure History } \\
\text { From patient to companian } \\
\text { From HCW to HCW } \\
\text { From patient to healthcare } \\
\text { staff }\end{array}$ & $\begin{array}{l}2 \\
4 \\
9 \\
9 \\
\end{array}$ & $\begin{array}{l}5.7 \\
11.4 \\
25.7 \\
25.7\end{array}$ \\
\hline $\begin{array}{l}\text { From patient to patient } \\
\text { From attendant to patient }\end{array}$ & 1 & 2.9 \\
\hline $\begin{array}{l}\text { PA Lung } x \text {-ray lesion } \\
\text { Yes } \\
\text { No } \\
\end{array}$ & $\begin{array}{l}21 \\
8 \\
\end{array}$ & $\begin{array}{l}60 \\
22.9 \\
\end{array}$ \\
\hline $\begin{array}{l}\text { Lesion in Tomography } \\
\text { Yes } \\
\text { No } \\
\end{array}$ & $\begin{array}{l}28 \\
1 \\
\end{array}$ & $\begin{array}{l}80 \\
2.9\end{array}$ \\
\hline $\begin{array}{l}\text { Survival } \\
\text { Death }\end{array}$ & $\begin{array}{l}25 \\
10\end{array}$ & $\begin{array}{ll}71 \\
28.6\end{array}$ \\
\hline
\end{tabular}

HCW: health Care Workers

neutrophil count, CRP and NLR values were found to be statistically related to survival (p:0.005, 0.001, 0.014 and 0.000, respectively) (Table 4). 
M. Gayaf ve ark., Clinical Characteristics and Transmission Routes of COVID-19 in the Early Period of the Pandemic in a Non-Covid Ward of a Chest Diseases Hospital

Table 2. Clinical characteristics of hospitalized patients and hospital staff with COVID-19.

\begin{tabular}{|c|c|c|c|}
\hline Characteristics & $\begin{array}{l}\text { Hospitalized patients } \\
\text { (n:16) }\end{array}$ & $\begin{array}{l}\text { Healthcare Staff } \\
\text { (n:19) }\end{array}$ & $\mathbf{P}$ \\
\hline $\begin{array}{l}\text { Gender } \\
\text { Male } \\
\text { Female } \\
\end{array}$ & $\begin{array}{l}7(43.8 \%) \\
9(56.3 \%) \\
\end{array}$ & $\begin{array}{c}4(21.1 \%) \\
15(78.9 \%)\end{array}$ & 0.282 \\
\hline $\begin{array}{l}\text { Comorbidity } \\
\text { Yes } \\
\text { No }\end{array}$ & $\begin{array}{c}14(87.5 \%) \\
2(12.5 \%) \\
\end{array}$ & $\begin{array}{c}4(21 \%) \\
15(79 \%)\end{array}$ & 0.001 \\
\hline $\begin{array}{l}\text { Hypertension } \\
\text { Yes } \\
\text { No } \\
\end{array}$ & $\begin{array}{c}5(31.3 \%) \\
11(68.7 \%) \\
\end{array}$ & $\begin{array}{c}1(5,3 \%) \\
18(94.7 \%) \\
\end{array}$ & 0.076 \\
\hline $\begin{array}{l}\text { Diabetes Mellitus } \\
\text { Yes } \\
\text { No }\end{array}$ & $\begin{array}{c}3(19 \%) \\
13(81 \%)\end{array}$ & $\begin{array}{c}0(0) \\
19(100)\end{array}$ & 0.092 \\
\hline $\begin{array}{l}\text { COPD } \\
\text { Yes } \\
\text { No } \\
\end{array}$ & $\begin{array}{c}6(38 \%) \\
10(62 \%) \\
\end{array}$ & $\begin{array}{c}0(0 \%) \\
19(100 \%) \\
\end{array}$ & 0.006 \\
\hline $\begin{array}{l}\text { Symptom } \\
\text { Yes } \\
\text { No }\end{array}$ & $\begin{array}{c}16(100 \%) \\
0(0 \%)\end{array}$ & $\begin{array}{c}13(68,4 \%) \\
6(31.6 \%)\end{array}$ & 0.103 \\
\hline $\begin{array}{l}\text { Fever } \geq 37.5 \\
\text { Yes } \\
\text { No } \\
\end{array}$ & $\begin{array}{l}9(56.3 \%) \\
7(43.8 \%) \\
\end{array}$ & $\begin{array}{c}7(36.9 \%) \\
12(63,1 \%) \\
\end{array}$ & 0.605 \\
\hline $\begin{array}{l}\text { Cough } \\
\text { Yes } \\
\text { No } \\
\end{array}$ & $\begin{array}{c}14(87,5 \%) \\
2(12,5 \%) \\
\end{array}$ & $\begin{array}{c}6(57,9 \%) \\
13(56.3 \%)\end{array}$ & 0.007 \\
\hline $\begin{array}{l}\text { Shortness of breath } \\
\text { Yes } \\
\text { No }\end{array}$ & $\begin{array}{c}13(81 \%) \\
3(19 \%) \\
\end{array}$ & $\begin{array}{c}2(11 \%)+4 \\
17(89 \%) \\
\end{array}$ & 0.000 \\
\hline $\begin{array}{l}\text { Lesion in lung x-ray } \\
\text { Yes } \\
\text { No }\end{array}$ & $\begin{array}{c}14(100 \%) \\
0(0 \%)\end{array}$ & $\begin{array}{l}7(46.7 \%) \\
8(53.3 \%)\end{array}$ & 0.002 \\
\hline $\begin{array}{l}\text { Lesion in CT } \\
\text { Yes } \\
\text { No } \\
\end{array}$ & $\begin{array}{c}13(100 \%) \\
0(0 \%) \\
\end{array}$ & $\begin{array}{c}10(66.7 \%) \\
5(33.3 \%) \\
\end{array}$ & 0.044 \\
\hline $\begin{array}{l}\text { Survival } \\
\text { Death }\end{array}$ & $\begin{array}{c}6(37.5 \%) \\
10(62.5 \%) \\
\end{array}$ & $\begin{array}{c}19(100 \%) \\
0(0 \%)\end{array}$ & 0.000 \\
\hline
\end{tabular}

\section{Disease Severity and Clinical and Laboratory Characteristics}

The neutrophil, NLR, leukocyte and CRP values were found to be statistically higher in patients with a severe disease condition (p:0.020, 0.037, $0.023,0.023$, respectively) (Table 5). As comorbidity, having COPD and involvement in lung $x$-ray were more in those with a severe condition (p:0.019,0.000,0.033, respectively) (Table 6).

\section{Potential "Super Spreaders"}

Based on exposure history, the nosocomial infection routes are given in Figure 1. It was found that there were 4 potential SARSCoV-2 spreaders ("Companion 01, Patient 01, Patient 02, and Patient 03”). During the hospitalization, a medical companion infected Patient 01. Patient 01,4 healthcare staff and a companion had positive exposure, and fever was detected in 3-8 days after exposure; and all 4 were confirmed to 
Table 3. Relation between patient characteristics and survival.

\begin{tabular}{|c|c|c|c|}
\hline \multirow{3}{*}{$\begin{array}{l}\text { Characteristics } \\
\text { Healthcare workers } \\
\text { Yes } \\
\text { No }\end{array}$} & \multicolumn{2}{|c|}{ Survival Status } & \multirow{3}{*}{$\begin{array}{l}\mathbf{P} \\
0,000\end{array}$} \\
\hline & \multirow{2}{*}{$\begin{array}{l}\text { Death } \\
\begin{array}{l}0(0 \%) \\
10(100 \%)\end{array}\end{array}$} & \multirow{2}{*}{$\begin{array}{l}\text { Survival } \\
19(76 \%) \\
6(24 \%)\end{array}$} & \\
\hline & & & \\
\hline $\begin{array}{l}\text { disease severity } \\
\text { Mild } \\
\text { Severe }\end{array}$ & $\begin{array}{c}0(0 \%) \\
10(100 \%)\end{array}$ & $\begin{array}{c}17(68 \%) \\
8(32 \%)\end{array}$ & 0,000 \\
\hline $\begin{array}{l}\text { Age } \\
<65 \\
\geq 65\end{array}$ & $\begin{array}{l}5(50 \%) \\
5(50 \%)\end{array}$ & $\begin{array}{c}22(88 \%) \\
3(12 \%)\end{array}$ & 0.027 \\
\hline $\begin{array}{l}\text { Gender } \\
\text { Female } \\
\text { Male }\end{array}$ & $\begin{array}{l}5(50 \%) \\
5(50 \%)\end{array}$ & $\begin{array}{c}19(76 \%) \\
6(24 \%)\end{array}$ & 0,227 \\
\hline $\begin{array}{l}\text { Comorbidity } \\
\text { Yes } \\
\text { No }\end{array}$ & $\begin{array}{l}9(90 \%) \\
1(10 \%)\end{array}$ & $\begin{array}{c}9(36 \%) \\
16(64 \%)\end{array}$ & 0,009 \\
\hline $\begin{array}{l}\text { COPD } \\
\text { Yes } \\
\text { No }\end{array}$ & $\begin{array}{l}4(40 \%) \\
6(60 \%)\end{array}$ & $\begin{array}{c}2(8 \%) \\
23(92 \%)\end{array}$ & 0.038 \\
\hline $\begin{array}{l}\text { Hypertension } \\
\text { Yes } \\
\text { No }\end{array}$ & $\begin{array}{l}3(33.3 \%) \\
6 \text { (66.7\%) }\end{array}$ & $\begin{array}{c}3(12 \%) \\
23(88 \%) \\
\end{array}$ & 0,314 \\
\hline $\begin{array}{l}\text { Symptom } \\
\text { No } \\
\text { Yes }\end{array}$ & $\begin{array}{c}0(0 \%) \\
10(100 \%)\end{array}$ & $\begin{array}{l}6(124 \%) \\
19(76 \%) \\
\end{array}$ & 0.289 \\
\hline $\begin{array}{l}\text { Shortness of breath } \\
\text { No } \\
\text { Yes }\end{array}$ & $\begin{array}{c}0(09 \%) \\
10(100 \%)\end{array}$ & $\begin{array}{c}16(64 \%) \\
9(36 \%)\end{array}$ & 0.000 \\
\hline $\begin{array}{l}\text { Lesion in lung } x \text {-ray } \\
\text { No } \\
\text { Yes }\end{array}$ & $\begin{array}{c}0(0 \%) \\
9(100 \%)\end{array}$ & $\begin{array}{c}8(40 \%) \\
12(60 \%)\end{array}$ & 0.033 \\
\hline $\begin{array}{l}\text { Involvement } \\
\text { in Tomography } \\
\text { No } \\
\text { Yes }\end{array}$ & $\begin{array}{c}0(0 \%) \\
8(100 \%)\end{array}$ & $\begin{array}{c}5(25 \%) \\
15(75 \%)\end{array}$ & 0,281 \\
\hline
\end{tabular}

have contracted SARS-CoV-2. Patient 02 infected two medical staff, and Patient 03 infected two patients. An HCW was infected from Patient 01 and Patient 02 (Figure 1).

The index case, who was a companion and initiated the spread, had a symptom of fever. Another index case was Patient 01, who had chronic renal failure and chronic obstructive pulmonary disease as a comorbid disease, and was pre-diagnosed with lung cancer. On the 10th day of hospitalization, it was observed that $s /$ he had fever and infiltration in lung x-ray 4 days after the transfer of the patient who was taken to a Non-Covid Ward. A Covid 19 PCR Test was done for Patient 01, and the diagnosis was confirmed. It was learned that the patient's companion (Companion 01) was admitted to another pandemic hospital with a Covid 19 infection.

Patient 02 was a 35-year-old female patient who lived in a nursing home with advanced 


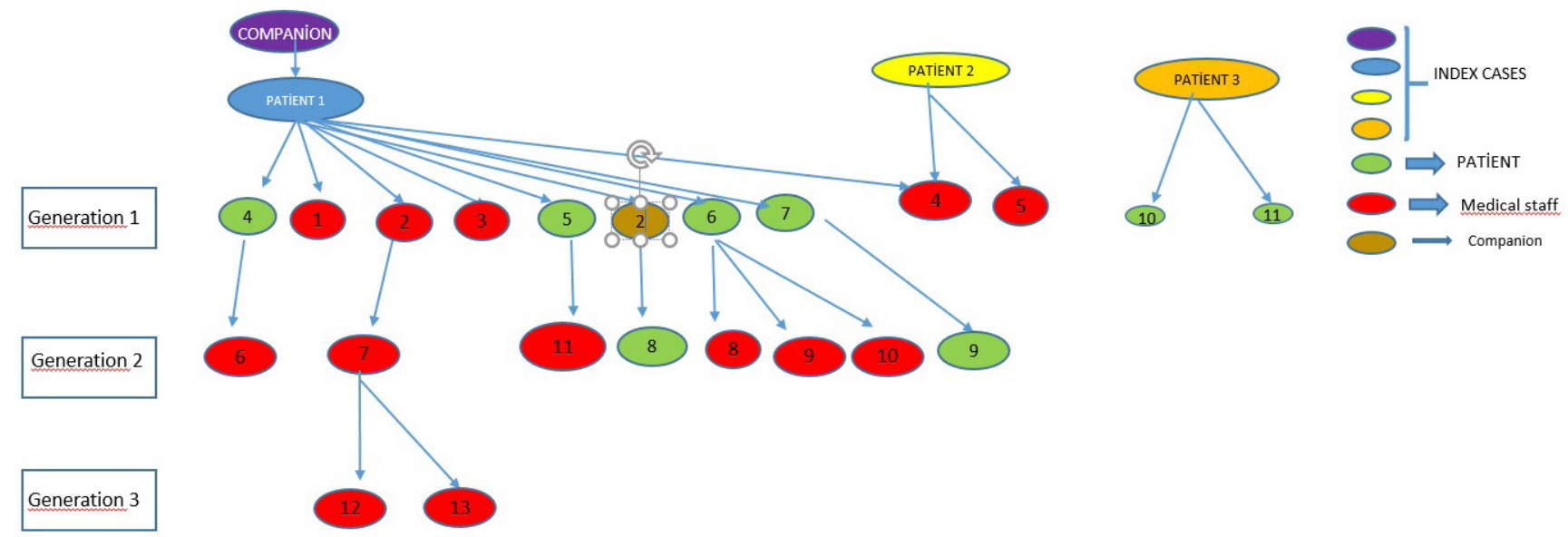

Figure 1. Transmission tree of Covid-19 outbreak.

Table 4. Relation between laboratory findings and survival of patients.

\begin{tabular}{|c|c|c|c|c|}
\hline $\begin{array}{l}\text { Laboratory } \\
\text { findings }\end{array}$ & Survival & Mean & $\begin{array}{l}\text { Standard } \\
\text { deviation }\end{array}$ & p \\
\hline \multirow[t]{2}{*}{ Hemoglobin } & Death & 10,76 & 1,52 & \multirow[t]{2}{*}{0,023} \\
\hline & Survival & 12,53 & 1,77 & \\
\hline \multirow[t]{2}{*}{ Neutrophil } & Death & 13962,50 & 9754,40 & \multirow[t]{2}{*}{0,001} \\
\hline & Survival & 2884,62 & 1478,09 & \\
\hline \multirow[t]{2}{*}{ Lymphocyte } & Death & 677,78 & 435,25 & \multirow[t]{2}{*}{0,005} \\
\hline & Survival & 1480,00 & 801,07 & \\
\hline \multirow[t]{2}{*}{ NLR } & Death & 26,50 & 20,41 & \multirow[t]{2}{*}{0,000} \\
\hline & Survival & 2,59 & 1,98 & \\
\hline \multirow[t]{2}{*}{ Leukocyte } & Death & 14355,56 & 9688,54 & \multirow[t]{2}{*}{0,006} \\
\hline & Survival & 5813,33 & 2903,66 & \\
\hline \multirow[t]{2}{*}{ Thrombocyte } & Death & 338,89 & 161,29 & \multirow[t]{2}{*}{0,493} \\
\hline & Survival & 271,20 & 118,17 & \\
\hline \multirow[t]{2}{*}{ CRP } & Death & 12.22 & 8.49 & \multirow[t]{2}{*}{0.014} \\
\hline & Survival & 4.22 & 6.27 & \\
\hline \multirow[t]{2}{*}{ D-Dimer } & Death & 2593,00 & 2315,06 & \multirow[t]{2}{*}{ NA } \\
\hline & Survival & 1208,07 & 2254,89 & \\
\hline \multirow[t]{2}{*}{ Ferritin } & Death & 870,88 & 864,50 & \multirow[t]{2}{*}{ NA } \\
\hline & Survival & 307,95 & 367,60 & \\
\hline
\end{tabular}

NLR: Neutrophil Lymphocyte Ratio CRP: C-reactive protein

mental retardation. She was hospitalized with pneumonia respiratory failure previously in another hospital and was referred to our hospital with pneumonia, pleurisy and respiratory failure.
Despite antibiotherapy, when there was ongoing fever and increased radiological infiltration, she was tested with a rapid antibody test kit, and a Covid 19 PCR test and the result was positive. The patient died on the 13th day of her hospitalization.

Patient 03 was a 35-year-old patient who was hospitalized with asthma and had comorbidities of Diabetes Mellitus, and pneumonia pleurisy. S/ he was diagnosed with Covid 19 with a PCR Test when she/he had a fever and radiologically bilateral lesions.

\section{DISCUSSION}

According to the report of ${ }^{(1)}$, as of 22.09.2020, there were 306,302 patients diagnosed with COVID-19, and the rate of mortality was $2.4 \%$. In our unit, the severe type accounted for $51.4 \%$ (18/35), and the rate of mortality was $28.6 \%$ (10/35), which was significantly higher than the reported results. In our study, there were 19 healthcare workers (10 nurses, 5 doctors, and 4 staff), 13 patients and 3 medical companions who were infected. Age, smoking history, and comorbidities might contribute to different outcomes of COVID-19 among patients and healthcare staff. It was found in our study that COPD is associated with severe COVID-19 and death at significant levels. 
Table 5. Relation between laboratory findings and disease severity of patients.

\begin{tabular}{|c|c|c|c|c|}
\hline \multirow{2}{*}{$\begin{array}{l}\text { Laboratory } \\
\text { findings } \\
\mathrm{Hgb}\end{array}$} & \multirow{2}{*}{$\begin{array}{l}\begin{array}{l}\text { Disease } \\
\text { severity }\end{array} \\
\text { Severe } \\
\end{array}$} & \multicolumn{3}{|c|}{ Mean \pm Standard deviation } \\
\hline & & 11,64 & 2,00 & \multirow[b]{2}{*}{0,198} \\
\hline & $\begin{array}{l}\text { Not } \\
\text { Severe }\end{array}$ & 12,31 & 1,59 & \\
\hline \multirow[t]{2}{*}{ Neutrophil } & Severe & 9221,43 & 9160,97 & \multirow[b]{2}{*}{0,020} \\
\hline & $\begin{array}{l}\text { Not } \\
\text { Severe }\end{array}$ & 2871,43 & 1863,43 & \\
\hline \multirow[t]{2}{*}{ Lymphocyte } & Severe & 1118,75 & 933,25 & \multirow[b]{2}{*}{0,133} \\
\hline & $\begin{array}{l}\text { Not } \\
\text { Severe }\end{array}$ & 1300,00 & 354,56 & \\
\hline \multirow[t]{2}{*}{ NLR } & Severe & 16,33 & 19,36 & \multirow[b]{2}{*}{0,037} \\
\hline & $\begin{array}{l}\text { Not } \\
\text { Severe }\end{array}$ & 2,43 & 1,79 & \\
\hline \multirow[t]{2}{*}{ WBC } & Severe & 11000,00 & 8408,33 & \multirow[b]{2}{*}{0,023} \\
\hline & $\begin{array}{l}\text { Not } \\
\text { Severe }\end{array}$ & 5050,00 & 2074,33 & \\
\hline \multirow[t]{2}{*}{ PLT } & Severe & 329,13 & 142,67 & \multirow[b]{2}{*}{0,098} \\
\hline & $\begin{array}{l}\text { Not } \\
\text { Severe }\end{array}$ & 231,50 & 102,29 & \\
\hline \multirow[t]{2}{*}{ CRP } & Severe & 8,71 & 7,76 & \multirow[b]{2}{*}{0,023} \\
\hline & $\begin{array}{l}\text { Not } \\
\text { Severe }\end{array}$ & 4,26 & 8,54 & \\
\hline \multirow[t]{2}{*}{ D-Dimer } & Severe & 1733,57 & 1863,50 & \multirow[b]{2}{*}{0,062} \\
\hline & $\begin{array}{l}\text { Not } \\
\text { Severe }\end{array}$ & 1542,00 & 3211,73 & \\
\hline \multirow[t]{2}{*}{ Ferritin } & Severe & 592,73 & 703,49 & \multirow[b]{2}{*}{ NA } \\
\hline & $\begin{array}{l}\text { Not } \\
\text { Severe }\end{array}$ & 356,96 & 500,33 & \\
\hline
\end{tabular}

HCWs have high risks in terms of acquiring infections in new disease outbreaks, particularly before transmission dynamics are identified. The cases reported in this study are among the first identified occupational transmission cases of SARS-CoV-2 to HCWs in Turkey. So far, little is known about SARS-CoV-2 transmission in healthcare institutions. It was reported from Illinois, Singapore, and Hong Kong that there were cohorts involving HCWs exposed to patients with COVID-19 without any documented HCP transmission ${ }^{(2-4)}$. Most HCW exposures among these were with patients and HCWs when using contact, droplet, or airborne precautions. While community transmission rates of COVID-19 increase, identifying if $\mathrm{HCW}$ infections are acquired at the workplace or in society becomes even more challenging. This examination brought with
Table 6. Relation between the characteristics of patients and disease severity.

\begin{tabular}{|c|c|c|c|}
\hline \multirow{3}{*}{$\begin{array}{l}\text { Characteristics } \\
\text { Healthcare staff } \\
\text { Yes } \\
\text { No } \\
\end{array}$} & \multicolumn{2}{|c|}{ Disease severity } & \multirow{3}{*}{$\begin{array}{l}\mathbf{p} \\
0.001\end{array}$} \\
\hline & Mild & Severe & \\
\hline & $\begin{array}{c}15(87,5 \%) \\
2(12.5 \%) \\
\end{array}$ & $\begin{array}{c}4(22.2 \%) \\
14(72.8 \%)\end{array}$ & \\
\hline $\begin{array}{l}\text { Age } \\
<65 \\
\geq 65\end{array}$ & $\begin{array}{c}17(100 \%) \\
0\end{array}$ & $\begin{array}{c}10(55.6 \%) \\
8(44.4 \%)\end{array}$ & 0.003 \\
\hline $\begin{array}{l}\text { Gender } \\
\text { Female } \\
\text { Male }\end{array}$ & $\begin{array}{c}14(82,3 \%) \\
3(18.8 \%)\end{array}$ & $\begin{array}{c}10(55.6 \%) \\
8(44.4 \%)\end{array}$ & 0.218 \\
\hline $\begin{array}{l}\text { Comorbidity } \\
\text { Yes } \\
\text { No }\end{array}$ & $\begin{array}{c}5(33.3 \%) \\
10(66.7 \%)\end{array}$ & $\begin{array}{c}13(72.2 \%) \\
7(35 \%)\end{array}$ & 0.060 \\
\hline $\begin{array}{l}\text { COPD } \\
\text { Yes } \\
\text { No } \\
\end{array}$ & $\begin{array}{c}0(0 \%) \\
17(100 \%)\end{array}$ & $\begin{array}{c}6(33.3 \%) \\
12(66.7 \%)\end{array}$ & 0.019 \\
\hline $\begin{array}{l}\text { Hypertension } \\
\text { Yes } \\
\text { No } \\
\end{array}$ & $\begin{array}{c}2(11.8 \%) \\
15(88.2 \%)\end{array}$ & $\begin{array}{c}4(22 \%) \\
14(78 \%)\end{array}$ & 0.659 \\
\hline $\begin{array}{l}\text { Symptom } \\
\text { No } \\
\text { Yes }\end{array}$ & $\begin{array}{c}6(35.3 \%) \\
11(64.7 \%)\end{array}$ & $\begin{array}{c}0(0 \%) \\
18(100 \%)\end{array}$ & 0.033 \\
\hline $\begin{array}{l}\text { Shortness of breath } \\
\text { No } \\
\text { Yes }\end{array}$ & $\begin{array}{c}12(70.6 \%) \\
5(29.4 \%)\end{array}$ & $\begin{array}{c}4(22.4 \%) \\
14(77.8 \%)\end{array}$ & 0.000 \\
\hline $\begin{array}{l}\text { Lesion in lung } x \text {-ray } \\
\text { No } \\
\text { Yes }\end{array}$ & $\begin{array}{l}7(58.3 \%) \\
5(41.7 \%)\end{array}$ & $\begin{array}{c}1(5.9 \%) \\
16(94.9 \%)\end{array}$ & 0.003 \\
\hline $\begin{array}{l}\text { Involvement in } \\
\text { tomography } \\
\text { No } \\
\text { Yes }\end{array}$ & $\begin{array}{l}4(33.3 \%) \\
8(66.7 \%)\end{array}$ & $\begin{array}{c}1(6.3 \%) \\
15(93.8 \%)\end{array}$ & 0.133 \\
\hline
\end{tabular}

it an opportunity for analyzing the exposure attributed to COVID-19 transmission in a healthcare institution without recognized community exposures. Identifying the exposures in HCWs that did and did not have COVID-19 might provide us with guidance on how to protect HCWs. Since patients at hospitals often contact inpatient caregivers and visitors who go in and out of the hospital often, they have a high risk of being infected, which exacerbates the infections of medical staff who are not in direct contact with the already-known infected cases. In addition, infected medical staff may not show any symptoms but be infectious. This might cause clustered infection in a unit. In our study, 25 of the infected cases $(76 \%)$ had a history of positive exposure, and 2 of them had a way of transmitting from patient to companion, 4 from a healthcare 
provider, 9 from patient to healthcare provider, 9 from patient to patient, and one case had transmission from companion to patient.

Age, history of smoking, and comorbid diseases might contribute to various outcomes of COVID-19 among patients and healthcare staff. In the present study, being 65 years of age or over, presence of additional disease, presence of COPD in history and presence of dyspnea as a symptom and involvement on chest radiograph were found to be significantly associated with survival. The fact that the patients had more comorbid diseases and respiratory failure than the HCWs, showed that they had a higher mortality rate. Additional diseases such as COPD affected the pulmonary function and dropped immunity, which suggests that SARS-CoV-2 infection is severe, and might cause poor outcomes in cases who have additional diseases, especially COPD. Liu et al. reported that CRP, LDH, and lymphocyte count might be used to predict the severity of lung injury ${ }^{(5)}$. In our study, the neutrophil, NLR, leukocyte and CRP values were higher in patients with a severe condition at statistically significant levels.

The main symptom of COVID-19 is fever, cough, fatigue, muscular soreness, chest depression, shortness of breath, diarrhea, and other symptoms ${ }^{(1)}$. The presence of the same symptoms in patients hospitalized with an acute attack of pneumonia or COPD in the early period of the epidemic created confusion. Many COVID19 cases had normal / reduced WBC counts and decreased lymphocyte counts. Early chest CTs of COVID-19 showed local ground-glass images and interstitial variations in the dorsal or peripheral parts of the lungs ${ }^{(6)}$. Also, multiple infiltrating shadows and lung consolidation might be detected in both lungs; and pleural effusion is seldom detected in severe patients ${ }^{(7,8)}$. Some of the initial COVID-19 patients might have one or multiple GGO-like variations, and this must be differentiated from lung cancer ${ }^{(9)}$. In some of our patients, there was no infiltration in PA lung $x$-rays in the first hospitalization however, infiltration in the lungs and fever were detected later. That was a clue for us; especially Patient 08, who was receiving radiotherapy due to lung cancer and had bone metastasis when fever and multiple infiltrations appeared. Upon the development of respiratory failure, s/he was taken to the Intensive Care Unit and died later.

SARS-CoV-2 is infectious at a high level and is susceptible to all populations. Respiration droplets and contact transmission are the main transmission routes as well as aerosol transmission (1). Even during the asymptomatic stage, an infected case is the main transmission source. Most of the patients hospitalized at chest diseases wards had cough complaints due to comorbidities (lung cancer, pneumonia, or COPD). The cough will cause a large number of droplets and aerosols in surrounding areas, and this might increase the efficacy of exposure. Therefore, COVID-19 patients are highly contagious in chest diseases departments. Moreover, the detection of patients in non-Covid wards in the early period of the outbreak, when the wards were being designed, increased the transmission. Due to insufficient preparation at the beginning of the pandemic, improper protective measures were applied at the initial stages of the pandemic. This resulted in a large number of infections for hospitalized patients and healthcare employees.

"Super Spreader" refers to a patient who transmits the infection potentially to more than 10 people. Retrospective analysis of 35 COVID19 cases in our unit showed 9 SARS-CoV-2 infected cases who had traceable exposure history with Companion 01 and Patient 02. In the other two index cases, Patient 02 infected only medical staff, and Patient 03 infected patients. In the four medical staff who were infected by Patients 01 and 02 , intensive care was needed and bilateral pneumonia was observed. However, there was no need for intensive care in other medical staff. We believe that this is probably related to the viral load the person encounters. A study has shown that viral loads peak in respiratory samples in the second week after the onset of the disease 
in mild group, but viral loads continued to be elevated in the third week in severe group. Virus duration was prolonged in patients who were older than 60 years and in males ${ }^{(10)}$. In another study (11), the authors' showed indicate that patients with severe COVID-19 tend to have a high viral load and a long virus-shedding period (similar to SARS in 2002-03), which suggests that the viral load of SARS-CoV-2 can be a marker to evaluate the severity and prognosis of the disease.

In summary, SARS-CoV-2 is highly infectious. Hospitalized patients and healthcare staff have high risks of infection in the pandemic. The fact that patients use the same toilets and bathrooms, share hospital rooms or have rooms close to each other, they need frequent use of respiratory treatments emitting aerosols, and some of them having medical companions make the transmission easier. Patients infected with COVID-19 have a high risk of death. Precautions in this respect include single hospitalization of suspected Covid19 patients; preparing quarantine wards in the proper form; and paying attention to hand hygiene, contact and using personal protective equipment (everybody in hospital, healthcare staff, patients, attendants, etc.). For this reason, applying comprehensive protective measures e.g. quarantine and disinfection are required in controlling the nosocomial infection.

For these reasons, especially at the beginning of the pandemic, it is very important to apply comprehensive preventive precautions such as quarantine and disinfection in controlling nosocomial infection in non-Covid services of the hospital.

Ethics Committee Approval: Approval was obtained from the Ethics Committee of Dr. Suat Seren Chest Diseases and Surgery Training and Research Hospital 27.05.2020/11).
Conflict of Interest: There is no conlict interest.

Funding: No financial support was received for the study.

Informed Consent: Consent of all patients was obtained.

\section{REFERENCES}

1. TC Sağlık Bakanlığı. Covid-19 Erişkin Hasta Tedavisi Accessed 15.5.2021.

2. Ghinai I, McPherson TD, Hunter JC, et al.; Illinois COVID19 Investigation Team. First known person-to-person transmission of severe acute respiratory syndrome coronavirus 2 (SARS-CoV-2) in the USA. Lancet 2020;395:1137-44 https://doi.org/10.1016/S0140-6736(20)30607-3

3. $\mathrm{Ng} \mathrm{K}$, Poon BH, KiatPuar TH, et al. COVID-19 and the risk to health care workers: a case report. Ann Intern Med 2020;172(11):766-7. https://doi.org/10.7326/L20-0175

4. Cheng VCC, Wong SC, Chen JHK, et al. Escalating infection control response to the rapidly evolving epidemiology of the coronavirus disease 2019 (COVID19) due to SARS-CoV-2 in Hong Kong. Infect Control Hosp Epidemiol. 2020;1-6. https://doi.org/10.1017/ice.2020.58

5. Liu YX, Yang Y, Zhang C, et al. Clinical and biochemical indexes from 2019-nCoV infected patients linked to viral loads and lung injury. Sci China Life Sci, 2020. https://doi.org/10.1007/s11427-020-1643-8

6. Ji JS, Wei TM, Yang WB, et al. CT early signs and differential diagnosis of new coronavirus pneumonia (COVID-19). Beijing: Science Press, 2020:7-15.

7. Song F, Shi N, Shan F, et al. Emerging Coronavirus 2019nCoV Pneumonia [published online ahead of print, 2020 Feb 6]. Radiology, 2020:200274. https://doi.org/10.1148/radiol.2020209021

8. Chung $M$, Bernheim A, Mei X, et al. CT Imaging Features of 2019 novel coronavirus (2019-nCoV) [published online ahead of print, 2020 Feb 4]. Radiology, 2020:200230 https://doi.org/10.1148/radiol.2020200230

9. Liu CY, Cai YX, Hao ZP, et al. Chest CT comparison of ground glass opacity (GGO)-like novel coronavirus (SARSCoV-2) infections and early-stage lung carcinoma. Chin J Clin Thoracic Cardiovascul Surg. (Chinese), 2020,27:376-380

10. Tianmin Xu, Cong Chen, Zhen Zhu, Manman Cui, Chunhua Chen, Hong Dai, Yuan Xue. Clinical features and dynamics of viral load in imported and non-imported patients with COVID-19. Int J Infect Dis 2020;94:68-71. https://doi.org/10.1016/j.ijid.2020.03.022

11. Liu Y, Yan LM, Wan L, Xiang TX, Le A, Liu JM, et al. Viral dynamics in mild and severe cases of COVID-19. Lancet Infect Dis. 2020 Jun; 20(6):656-57. https://doi.org/10.1016/S1473-3099(20)30232-2 